\title{
CHARACTERIZATION OF THE EXPERT VEHICLE CONTROL SURFACES BY MEANS OF IR MEASUREMENTS
}

\section{Gennaro Cardone* and Antonio Del Vecchio ${ }^{+}$}

\author{
*University of Naples"Federico II", DETEC Department, p.le Tecchio, 80127 Naples (Italy), \\ email: gcardone@unina.it \\ ${ }^{+}$CIRA - Italian Center for Aersopace Research - Via Maiorise, 81043 Capua (Italy), \\ email: a.delvecchio@cira.it
}

The aim of the present work is to designs the IR tests to be performed in the CIRA plasma wind tunnel "Scirocco" in the frame of technology project CLAE. The objective of this project is the characterization of the EXPERT vehicle control surfaces. In particular, model configuration and plasma test conditions have been selected in order to duplicate either the shock wave boundary layer interaction occurring around the body-flap, either the associated mechanical and thermal loads on the control device encountered in some EXPERT capsule re-entry flight conditions. Within this Project frame, at first, two tests on the same model at two different aero-thermodynamic conditions have been planned. These tests are focused on the aero-thermodynamic behaviour of the flow on the control device. In a subsequent phase other tests are foreseen to analyse the different materials of the control surface behaviour.

The SCIROCCO arc-jet facility presents some peculiarities that make it unique in the world. In particular, the arc heater length and diameter are respectively of $5500 \mathrm{~mm}$ and $110 \mathrm{~mm}$ and its maximum power is $70 \mathrm{MW}$. Briefly, the SCIROCCO facility is a typical segmented constrictor Arc-Jet Wind Tunnel (AWT). The gas used for the tests is dry compressed air with mass flow variable from 0.1 to $3.5 \mathrm{~kg} / \mathrm{sec}$. It accelerates through a convergent-divergent conical nozzle with interchangeable exit diameters up to $1950 \mathrm{~mm}$. The flow velocity at the nozzle exit can reach a value of $7000 \mathrm{~m} / \mathrm{s}$. A powerful Vacuum System working via the act ion of twelve steam ejectors is located downstream of the test chamber. During a test, after the stable flow condition is, in the test chamber, attained and confirmed by probe sensor, $t$ he testing model is put into the flow by the support system with characteristic time of 2-5sec. The thermocamera used is the Agema Thermovision THV900LW. It is equipped with a Cd-Te-Hg detector scanning and its field of view holds in the IR waveband 8-12 $\mu \mathrm{m}$ with a frame of 136 not-interlaced lines in $1 / 15 \mathrm{~s}$. The thermal image is digitized with a resolution of $272 \times 136$ pixels that, in combination to a $10^{\circ} \times 20^{\circ} \mathrm{IR}$ lens (or $5^{\circ} \times 10^{\circ}$ ) placed at the distance of about $4 \mathrm{~m}$ from the test sample, results in a spatial resolution of about $1 \mathrm{~cm} / \mathrm{pixel}(0.5 \mathrm{~cm} / \mathrm{pixel})$. The thermocamera is mounted in a protected box on a robotized remotely controlled gear, in order to avoid any disturbance from ambient radiation. In front of the thermo camera a ZnSe IR windows with a special coating to reduce the band pass wavelength region to the proper values of about 8-10mm is installed.

The model geometry that will be tested is show in fig. 1 . The leading edge is a water cooled copper alloy cylinder. The upper part of the model is covered by a metallic panel of PM1000, equipped of pressure and heat flux sensors (RAFLEX) along the surface. A flap covered by a $\mathrm{C} / \mathrm{SiC}$ panel is installed with an angle of $20^{\circ}$ with respect to the PM1000 panel, in order to deflect the flow of $20^{\circ}$ 


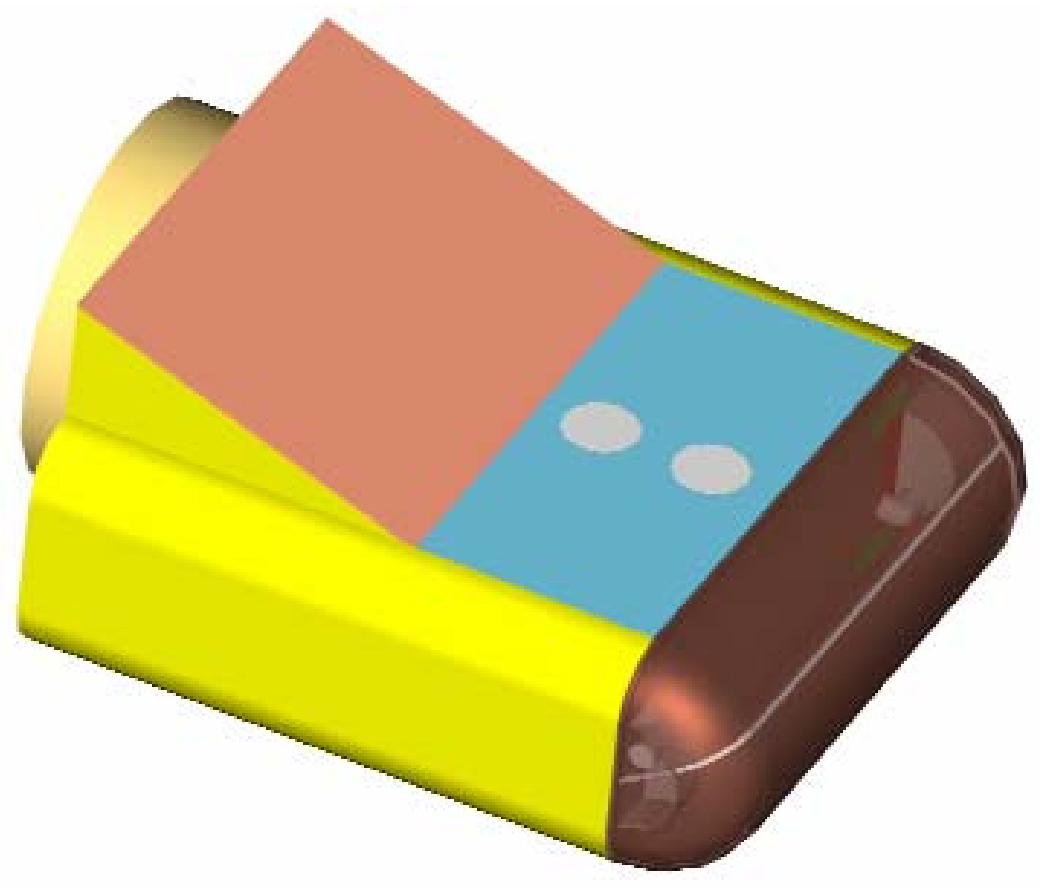

Fig. 1 Model geometry

Such a model is conceived to be applied for several types of tests. All the components of the test article are easily removable, in order to be replaced by others, for the same scope or different. Those characteristics make such a model to be very flexible for different types of applications. This is a very important aspect of the test article that will not be used only for the scope of this work, but also for other applications, very different from this one.

In order to obtain information of flow field around the control surface (size of the re-circulation induced by the shock wave boundary layer interaction, peak heating position, re-attachment front of the boundary layer etc.) the time sequence of temperature map that will be acquired during the tests will be processed be means of ad hoc developed heat flux sensor (based on classical thin film sensor) but taking in account the 3D model effects. The IR tests will be also used to evaluate and understand the effects of different surface material properties (catalysis, emissivity) on the flow past the flap. IR measurements will be also compared with heat flux measurements performed with standard heat-flux sensors and thermocouples. 